\title{
Differential associations between retinal signs and CMBs by location
}

The AGES-Reykjavik Study

Chengxuan Qiu, PhD,* Jie Ding, PhD,* Sigurdur Sigurdsson, MSc, Diana E. Fisher, MA, Qian Zhang, MSc, Gudny Eiriksdottir, MSc, Ronald Klein, MD, Mark A. van Buchem, MD, PhD, Vilmundur Gudnason, MD, PhD, Mary Frances Cotch, PhD, and Lenore J. Launer, PhD

Neurology ${ }^{\circledast}$ 2018;90:e142-148. doi:10.1212/WNL.0000000000004792

\author{
Correspondence \\ Dr. Launer \\ LaunerL@nia.nih.gov \\ or Dr. Qiu \\ chengxuan.qiu@ki.se
}

\begin{abstract}
Objective

To test the hypothesis that age-related macular degeneration (AMD) and retinal microvascular signs are differentially associated with lobar and deep cerebral microbleeds (CMBs).

\section{Methods}

CMBs in lobar regions indicate cerebral amyloid angiopathy (CAA). $\beta$-Amyloid deposits are implicated in both CAA and AMD. Deep CMBs are associated with hypertension, a major risk factor for retinal microvascular damage. This population-based cohort study included 2,502 participants in the Age, Gene/Environment Susceptibility (AGES)-Reykjavik Study who undertook binocular digital retinal photographs at baseline (2002-2006) to assess retinal microvascular signs and AMD and brain MRI scan at both baseline and follow-up (2007-2011) to assess CMBs. We assessed retinal microvascular lesion burden by counting the 3 retinal microvascular signs (focal arteriolar narrowing, arteriovenous nicking, and retinopathy) concurrently present in the participant. We used multiple logistic models to examine the association of baseline retinal pathology to incident $\mathrm{CMBs}$ detected at follow-up.
\end{abstract}

\section{Results}

During an average 5.2 years of follow-up, 461 people (18.3\%) developed new CMBs, including 293 in exclusively lobar regions and 168 in deep regions. Pure geographic atrophy was significantly associated with strictly lobar CMBs (multivariable-adjusted odds ratio $2.59,95 \%$ confidence interval $[\mathrm{CI}] 1.01-6.65$ ) but not with deep CMBs. Concurrently having $\geq 2$ retinal microvascular signs was associated with a 3-fold (95\% CI 1.73-5.20) increased likelihood for deep CMBs but not exclusively lobar CMBs.

\section{Conclusions}

Retinal microvascular signs and pure geographic atrophy may be associated with deep and exclusively lobar CMBs, respectively, in older people. These results have implications for further research to define the role of small vessel disease in cognitive impairment.

*These authors contributed equally to this work.

From the Intramural Research Program (C.Q., J.D., Q.Z., L.J.L.), Laboratory of Epidemiology and Population Sciences, National Institute on Aging, NIH, Bethesda, MD; Aging Research Center (C.Q.), Department of Neurobiology, Care Sciences and Society, Karolinska Institutet-Stockholm University, Sweden; Icelandic Heart Association (S.S., G.E., V.G.), Kopavogur; Division of Epidemiology and Clinical Research (D.E.F., M.F.C.), National Eye Institute, NIH, Bethesda, MD; Ophthalmology and Visual Sciences (R.K.), University of Wisconsin Madison; Department of Radiology (M.A.v.B.), Leiden University Medical Centre, the Netherlands; and Faculty of Medicine (V.G.), University of Iceland, Reykjavik.

Go to Neurology.org/N for full disclosures. Funding information and disclosures deemed relevant by the authors, if any, are provided at the end of the article.

The Article Processing Charge was funded by Swedish Research Council.

This is an open access article distributed under the terms of the Creative Commons Attribution License 4.0 (CC BY), which permits unrestricted use, distribution, and reproduction in any medium, provided the original work is properly cited. 


\section{Glossary}

AD = Alzheimer disease; AGES = Age, Gene/Environment Susceptibility; AMD = age-related macular degeneration; BMI = body mass index; $\mathbf{C A A}=$ cerebral amyloid angiopathy; $\mathbf{C M B}=$ cerebral microbleed; $\mathrm{SVD}=$ small vessel disease; $\mathbf{W M L}=$ white matter lesion.

Cerebral microbleeds (CMBs) are a neuroimaging marker of cerebral small vessel disease (SVD) and are associated with stroke, cognitive decline, and dementia. ${ }^{1-3}$ Histopathologically, CMBs on $\mathrm{T} 2^{*}$-weighted MRI correspond to small perivascular hemosiderin deposits. ${ }^{1}$ It has been assumed that CMBs in the deep or infratentorial areas reflect hypertensive microangiopathy, whereas lobar CMBs represent a marker for cerebral amyloid angiopathy (CAA). ${ }^{1,4}$

Microvasculature in the retina and the brain shares embryologic origin, anatomic features, metabolic activities, and similar patterns of vascularization and extracellular deposits. ${ }^{5}$ Retinal microvascular signs (e.g., focal arteriolar narrowing and arteriovenous nicking) are thought to result partly from short- or long-term exposures to cardiovascular risk factors, especially hypertension. ${ }^{6}$ Population-based cohort studies have related retinal microvascular signs to white matter lesions (WMLs), lacunar infarcts, and subcortical infarcts. ${ }^{7-9}$ However, the longitudinal association of retinal microvascular pathology with CMBs by location has yet to be examined.

Age-related macular degeneration (AMD) is a common retinal degenerative disorder among older people. Evidence has emerged that $\beta$-amyloid deposits, a hallmark of Alzheimer disease (AD), may be involved in the pathogenesis of AMD. ${ }^{10,11}$ Thus, retinal amyloid plaque has been proposed as a potential biomarker for $\mathrm{AD}$ and amyloid deposits in the brain. ${ }^{12-14}$

The cross-sectional data from the population-based Age, Gene/Environment Susceptibility (AGES)-Reykjavik Study showed an association of CMBs with retinal microvascular signs ${ }^{15}$ and a suggestive association with pure geographic atrophy. ${ }^{16}$ In this follow-up study, we seek to investigate the longitudinal associations of retinal microvascular signs and AMD with CMBs at specific locations in the brain. We hypothesize that the load of retinal microvascular signs is associated specifically with deep CMBs due primarily to hypertensive microangiopathy, whereas $\mathrm{AMD}$ is associated with lobar CMBs due predominantly to CAA.

\section{Methods}

\section{Study population}

This is a longitudinal population-based cohort study. Participants were from the AGES-Reykjavik Study that aimed at investigating genetic and environmental factors contributing to clinical and subclinical diseases in aging, as fully described elsewhere. ${ }^{17}$ Briefly, the Reykjavik Study was launched in 1967 by the Icelandic Heart Association and included a cohort of men and women born from 1907 to 1935 . In 2002 to 2006, survivors of the cohort were invited to participate in the AGESReykjavik Study, and 5,764 persons were examined (baseline, AGES-I). In 2007 to 2011, survivors of the AGES-I cohort were invited for follow-up examination, and 3,316 were reassessed (follow-up, AGES-II). In total, 2,672 persons underwent brain MRI scans in both AGES-I and AGES-II and had the brain images needed for assessment of CMBs. ${ }^{18}$ Of these, we excluded 133 persons who had no data of either AMD or retinal vascular signs and additional 37 individuals who were diagnosed with prevalent dementia at baseline, leaving 2,502 persons for the present analysis. Of these, data on AMD and retinal microvascular signs were available in 2,484 and 2,497 persons, respectively.

\section{Standard protocol approvals, registrations, and patient consents}

The AGES-Reykjavik Study was approved by the Icelandic National Bioethics Committee (VSN-00-063), which acts as the Institutional Review Board of the Icelandic Heart Association, and by the Institutional Review Board for the National Institute on Aging, NIH. Written informed consent was obtained from all participants.

\section{Fundus photography and assessments}

Digital fundus photographs were taken at baseline (2002-2006) following standardized protocols. ${ }^{19}$ Briefly, after the maximal pharmacologic dilation of the pupils, two $45^{\circ}$ retinal images, centered on the optic disc and the fovea, were taken of each eye with a 6.3-megapixel Canon digital nonmydriatic camera (Canon, Lake Success, NY). EyeQ Lite software (an image-processing database for storage, retrieval, and manipulation of digital retinal images) was used for retinal image acquisition, assessment, and archiving.

Using the modified Wisconsin Age-Related Maculopathy Grading scheme, graders masked to participants' identity evaluated digital retinal images at the University of Wisconsin Ocular Epidemiology Reading Center for AMD lesions. ${ }^{19}$ Early AMD was defined by the presence of any soft drusen and pigmentary abnormalities or the presence of large soft drusen. Late AMD was defined by the presence of either geographic atrophy or signs of exudative AMD (i.e., subretinal hemorrhage, subretinal fibrous scar, retinal pigment epithelial detachment, sensory retinal detachment, and any signs of treatment for neovascular AMD). Reassessments on randomly selected images of 25 eyes showed excellent intraobserver $(\kappa=0.82-1.0)$ and interobserver $(\kappa=0.88-1.0)$ agreement on AMD classification. ${ }^{19}$

Retinal microvascular signs were assessed following a validated protocol..$^{20}$ Retinal focal arteriolar narrowing or arteriovenous 
nicking was defined as present if the grader was at least $90 \%$ certain that the lesion was present in a given eye. Retinopathy lesions, classified similarly, included retinal blot hemorrhages, microaneurysms, soft exudates, and more advanced lesions associated with diabetic retinopathy. The intraobserver and interobserver agreement was fair to good $(\kappa=0.31-0.76)$ for focal arteriolar signs and excellent $(\kappa=0.81-1.00)$ for retinopathy lesions. ${ }^{21}$

\section{MRI acquisition and reading protocol}

All eligible participants had high-resolution brain MRI acquired on a 1.5T Signa TwinSpeed System (General Electric Medical Systems, Waukesha, WI) at both the AGES-I and AGES-II examinations. ${ }^{18,22}$ Briefly, the core protocol included a T1-weighted 3-dimensional spoiled gradient-echo sequence, a proton density/T2-weighted fast spin-echo sequence, a fluidattenuated inversion recovery sequence, and a $\mathrm{T} 2{ }^{*}$-weighted gradient-recalled echo sequence. MRI scan parameters for all sequences are fully reported elsewhere. ${ }^{15,22}$ All images were acquired to give full brain coverage, and slices were angled parallel to the anterior commissure-posterior commissure line to give reproducible image views in the oblique-axial plane.

All brain images were analyzed automatically with the AGES/ Montreal Neurological Institute image postprocessing pipeline, ${ }^{22}$ which segments the whole brain into gray matter, normal white matter, WMLs, and CSF. Brain infarcts were defined as defects in the brain parenchyma with a signal intensity that was isointense to that of CSF on all sequences with a diameter $\geq 4 \mathrm{~mm}$, except for infarcts in the cerebellum, brainstem, and cortex, which had no size criteria. ${ }^{21}$

\section{Assessment and categorization of CMBs}

CMBs were defined as a focal area of signal void within the brain parenchyma that is visible on the $\mathrm{T} 2{ }^{*}$-weighted gradientrecalled echo images and smaller or invisible on T2-weighted images. The semiquantitative assessment of CMBs is conducted at a computer workstation with customized in-house software for the longitudinal image analysis. To maximize comparability of baseline and follow-up images, they were aligned with each other with a linear coregistration and viewed side by side on the computer screen. This involved registering both the baseline and follow-up proton density/T2-weighted, $\mathrm{T} 2{ }^{*}$-weighted, and fluid-attenuated inversion recovery images for each participant to the baseline T1-weighted 3-dimensional spoiled gradient-echo images because the T1s had the highest spatial resolution of all images in the protocol. By performing this coregistration, we removed differences in image slice alignments due to different positioning of the participant in the head coil and different positioning of slices by the operator of the MRI scanner at the time of scanning in the 2 visits. The follow-up MRI scans were read by investigators blinded to the baseline scans. If a CMB was found on the follow-up MRI scan, the baseline MRI scan was examined to determine whether the $\mathrm{CMB}$ was present in the same location of the slice. If so, the follow-up CMB was labeled prevalent; if not, the CMB was labeled incident. This image preprocessing and rating procedure made it possible to reliably distinguish incident $\mathrm{CMBs}$ from prevalent CMBs. Both the intrarater agreement and interrater agreement based on 2 ratings within a 6-month interval were good ( $\kappa$ range $0.70-0.75) .{ }^{18}$

CMB location was recorded for lobar (frontal, temporal, parietal, and occipital) and deep or infratentorial (basal ganglia, thalamus, corpus callosum, brainstem, and cerebellum) regions. Because we were interested in CMBs with an amyloid (CAA) or vascular (hypertensive microangiopathy) origin according to our hypothesis and because it is possible that lobar CMBs are also related to hypertension, we classified people with CMBs by location as follows: people with at least 1 new $\mathrm{CMB}$ restricted to lobar regions were considered to have lobar CMBs exclusively, and those with CMBs in a deep or infratentorial region with or without concomitant lobar CMBs were classified as having deep CMBs. ${ }^{2,18}$

\section{Potential confounders}

Demographics, cardiovascular risk factors, ischemic cerebral SVD, and APOE genotype at baseline may confound the association between retinal signs and incident CMBs. Age, sex, education, and smoking were assessed via questionnaire administered by a trained interviewer. ${ }^{17}$ Body mass index (BMI) was calculated from measured weight (kilograms) divided by height (meters) squared. Fasting blood glucose and total cholesterol were measured according to standard protocols. Use of medications (e.g., antihypertensive and hypoglycemic agents) was ascertained from medication vials brought to the study center. Arterial blood pressure was measured twice in a sitting position to the nearest $2 \mathrm{~mm} \mathrm{Hg}$ with a mercury sphygmomanometer with a standard-sized cuff. Hypertension was defined as having a self-reported history of hypertension, having a blood pressure $\geq 140 / 90 \mathrm{~mm} \mathrm{Hg}$, or using antihypertensive drugs. Diabetes mellitus was defined according to self-reported history of diabetes mellitus, use of hypoglycemic drugs, insulin injection, or fasting blood glucose level $\geq 7.0 \mathrm{mmol} / \mathrm{L}$. $^{15}$ APOE genotype was determined with standard DNA amplification and restriction isotyping. ${ }^{23}$

\section{Statistical analysis}

Baseline characteristics of participants were presented by incident CMBs detected at follow-up. General linear or logistic regression analysis was performed to compare means or proportions with adjustment for age and sex. To assess the extensity and severity of retinal vascular lesions, we created an index for retinal microvascular burden by counting the 3 retinal microvascular signs concurrently present in the participant (range 0-3), i.e., retinal focal arteriolar narrowing, arteriovenous nicking, and retinopathy lesions. ${ }^{24}$ The index was categorized into 0 (reference), 1 , and $\geq 2$; we combined the index scores of 2 and 3 because of the low number of participants who had a score of $3(n=9)$. We used binomial logistic models to estimate the odds ratio and $95 \%$ confidence interval of incident $\mathrm{CMBs}$ associated with $\mathrm{AMD}$ and the index of retinal microvascular signs. Multinomial logistic regression models were constructed to examine the association between retinal 
signs and CMBs by location (deep vs strictly lobar areas)., ${ }^{2,18}$ We reported the main results from the model that was adjusted for age, sex, types of coil, follow-up interval, current smoking, BMI, systolic blood pressure, use of antihypertensive medications, total cholesterol, diabetes mellitus, MRI markers of cerebral SVD (i.e., subcortical infarcts, volume of WMLs, and prevalent $\mathrm{CMBs}$ ) assessed at baseline, and APOE $\varepsilon 4$ allele. Stata Release 12 (StataCorp 2011, StataCorp LP, College Station, TX) was used for all analyses.

\section{Results}

\section{Baseline characteristics of participants}

At baseline, the mean age of the 2,502 participants was 74.6 $(\mathrm{SD}=4.7)$ years, and $58.63 \%$ were women. After an average $5.2(\mathrm{SD}=0.2)$ years of follow-up, new CMBs were detected in 461 (18.43\%) persons; of these, $293(63.56 \%)$ had CMBs exclusively in lobar regions and 168 (36.44\%) in deep brain regions. Compared with persons who did not develop new CMBs at follow-up, those who developed incident CMBs were older; were more likely to be male, smoke, and have diabetes mellitus; and had a lower BMI, a higher level of diastolic blood pressure, and a larger volume of WMLs, subcortical infarcts, and prevalent CMBs at baseline (all $p<0.05$ ), but the 2 groups did not differ significantly in educational attainments, systolic blood pressure, hypertension, use of antihypertensive drugs, total cholesterol, and APOE $\varepsilon 4$ allele (table 1).

\section{AMD subtypes and CMBs by anatomic location}

At baseline, of the 2,484 persons, early AMD was detected in $451(18.16 \%)$ and late AMD in 92 (3.70\%); 63 (2.54\%) of those with late AMD had exudative AMD, and 29 (1.12\%) had pure geographic atrophy. After adjustment for multiple potential confounders, including baseline MRI markers of cerebral SVD (e.g., prevalent CMBs, subcortical infarcts, and volume of WMLs), early AMD was not significantly associated with global or regional incident CMBs (table 2). However, pure geographic atrophy, but not exudative AMD, was significantly associated with an $\approx 2.5$-fold increased likelihood of developing new CMBs exclusively in the lobar areas. Neither exudative AMD nor pure geographic atrophy was significantly associated with deep CMBs (table 2).

\section{Retinal microvascular signs and CMBs by anatomic location}

After controlling for multiple potential confounders, including baseline imaging markers of cerebral SVD, having retinal focal arteriolar narrowing and arteriovenous nicking was significantly associated with an increased likelihood of developing new CMBs specifically in the deep brain areas, not exclusively in the lobar regions (table 3). The presence of retinopathy

Table 1 Baseline characteristics of study participants by incident CMBs detected at the follow-up examination

\begin{tabular}{|c|c|c|c|c|}
\hline \multirow[b]{2}{*}{ Characteristics } & \multirow[b]{2}{*}{ Total sample $(n=2,502)^{a}$} & \multicolumn{3}{|c|}{ Incident CMBs detected at follow-up } \\
\hline & & No $(n=2,041)$ & Yes $(n=461)$ & $p$ Value $^{\mathrm{b}}$ \\
\hline Age, y & $74.6(4.7)$ & $74.4(4.7)$ & $75.3(5.0)$ & 0.001 \\
\hline Female, n (\%) & $1,467(58.6)$ & $1,233(60.4)$ & $234(50.8)$ & $<0.001$ \\
\hline Elementary school, n (\%) & 486 (19.5) & $401(19.7)$ & $85(18.5)$ & 0.656 \\
\hline Current smoking, n (\%) & $273(10.9)$ & $205(10.0)$ & $68(14.8)$ & 0.001 \\
\hline $\mathrm{BMI}, \mathrm{kg} / \mathrm{m}^{2}$ & $27.2(4.1)$ & $27.3(4.2)$ & $26.8(4.0)$ & 0.038 \\
\hline Diabetes mellitus, n (\%) & $230(9.2)$ & $174(8.5)$ & $56(12.2)$ & 0.029 \\
\hline Hypertension, n (\%) & $1,933(77.3)$ & $1,571(77.0)$ & $362(78.5)$ & 0.720 \\
\hline Systolic pressure, mm Hg & $141.1(19.7)$ & $140.6(19.0)$ & $143.0(19.8)$ & 0.106 \\
\hline Diastolic pressure, mm Hg & $74.2(9.3)$ & $74.0(9.1)$ & $75.4(9.9)$ & 0.011 \\
\hline Use of antihypertensive drugs, $n$ (\%) & $1,505(60.2)$ & $1,228(60.2)$ & $277(60.1)$ & 0.826 \\
\hline Total cholesterol, $\mathrm{mmol} / \mathrm{L}$ & $5.65(1.13)$ & $5.67(1.13)$ & $5.59(1.13)$ & 0.862 \\
\hline Total volume of WMLs, $\mathrm{mL}$ & $11.7(6.6-21.8)$ & $11.0(6.3-20.0)$ & $17.0(9.1-29.8)$ & $<0.001$ \\
\hline Subcortical infarcts & $185(7.4)$ & $133(6.5)$ & $52(11.3)$ & 0.004 \\
\hline Prevalent CMBs, n (\%) & $426(17.0)$ & $296(14.5)$ & $130(28.2)$ & $<0.001$ \\
\hline APOE \&4 allele, $\mathrm{n}(\%)$ & $648(26.0)$ & $513(25.2)$ & $135(29.3)$ & 0.060 \\
\hline
\end{tabular}

Abbreviations: $\mathrm{BMI}=$ body mass index; $\mathrm{CMB}=$ cerebral microbleed; $\mathrm{WML}=$ white matter lesion.

Values are mean (SD) or $\mathrm{n}(\%)$, except total volume of WMLs, which is median (interquartile range).

${ }^{a}$ This number included participants with measurement of either age-related macular degeneration $(n=2,484)$ or retinal microvascular signs $(n=2,497)$.

${ }^{\mathrm{b}} P$ value was adjusted for age and sex except the test of age or sex difference. 
Table 2 Association between AMD and incident CMBs by anatomic location $(n=2,484)$

\begin{tabular}{|c|c|c|c|c|c|c|}
\hline \multirow[b]{2}{*}{ AMD } & \multicolumn{2}{|c|}{ Global CMBs $(n=456)$} & \multicolumn{2}{|c|}{ Exclusively lobar CMBs $(n=290)^{a}$} & \multicolumn{2}{|c|}{ Deep CMBs $(n=166)^{a}$} \\
\hline & No. & Odds ratio $(95 \% \mathrm{Cl})^{\mathrm{b}}$ & No. & Odds ratio $(95 \% \mathrm{Cl})^{\mathrm{b}}$ & No. & Odds ratio $(95 \% \mathrm{Cl})^{\mathrm{b}}$ \\
\hline No AMD $(n=1,941)$ & 352 & 1.00 (Reference) & 219 & 1.00 (Reference) & 133 & 1.00 (Reference) \\
\hline Early AMD $(n=451)$ & 84 & $0.90(0.68-1.20)$ & 54 & $0.96(0.68-1.34)$ & 30 & $0.82(0.53-1.26)$ \\
\hline Late AMD $(n=92)$ & 20 & $0.95(0.55-1.67)$ & 17 & $1.40(0.76-2.56)$ & 3 & $0.34(0.10-1.16)$ \\
\hline Exudative AMD ( $n=63)$ & 11 & $0.70(0.34-1.45)$ & 10 & $1.11(0.52-2.40)$ & 1 & $0.16(0.02-1.19)$ \\
\hline Pure geographic atrophy $(n=29)$ & 9 & $1.91(0.80-4.56)$ & 7 & $2.59(1.01-6.65)$ & 2 & $0.96(0.20-4.49)$ \\
\hline
\end{tabular}

Abbreviations: $\mathrm{AMD}$ = age-related macular degeneration; $\mathrm{Cl}$ = confidence interval; $\mathrm{CMB}=$ cerebral microbleed.

a Participants with no incident CMBs $(n=2,028)$ were held as a referent group for multinomial logistic modeling when the associations with incident CMBs by anatomic location (i.e., exclusively lobar or deep CMBs vs no CMBs) were examined.

${ }^{b}$ Odds ratio $(95 \% \mathrm{Cl})$ was adjusted for age, sex, types of coils, follow-up interval, current smoking, systolic blood pressure, use of antihypertensive drugs, body mass index, diabetes mellitus, total cholesterol, baseline prevalent CMBs, subcortical infarcts, volume of white matter lesions, and APOE 44 allele.

lesions was significantly associated with an increased likelihood of having global CMBs. When the 3 retinal microvascular signs were aggregated into a composite index, we found that, compared to persons having none of the 3 retinal microvascular signs, each additional lesion was associated with a significantly increased likelihood of developing new CMBs globally ( $p$ for trend $=0.047$ ), especially in deep brain regions ( $p$ for trend $<0.001)$; concurrently possessing $\geq 2$ retinal microvascular signs was significantly associated with a 3 -fold increased likelihood of developing deep CMBs, whereas there was no significant association between the index of retinal microvascular signs and the likelihood of having strictly lobar CMBs (table 3 ).

\section{Additional analyses}

First, we repeated the analyses by excluding persons with prevalent CMBs at baseline $(n=427)$, which yielded results similar to those reported in tables 2 and 3 (table e-1, http://links.lww.com/WNL/A18). Furthermore, there was no significant association between AMD and exclusively deep $\mathrm{CMBs}$, and the associations of retinal microvascular signs (individual signs or the composite index) with exclusively deep $\mathrm{CMBs}$ were less evident compared to those associations with the presence of any deep CMBs (data not shown). Finally, there was no statistical interaction of $A P O E \& 4$ allele with either retinal vascular signs or $\mathrm{AMD}$ for incident $\mathrm{CMBs}$, and analysis stratified by APOE $\varepsilon 4$ did not show any additional significant associations between retinal signs and incident $\mathrm{CMBs}$ (data not shown).

\section{Discussion}

In a well-established cohort of older adults, we found evidence suggesting that pure geographic atrophy might be associated with an increased risk of developing CMBs exclusively in lobar areas but not in deep brain regions. Early AMD and exudative AMD were not associated with developing CMBs. In contrast, a greater number of retinal microvascular signs was associated with a higher likelihood of developing new CMBs in deep brain regions and not with CMBs only in lobar areas. The observed associations are present even after controlling for demographics, cardiovascular risk factors, $A P O E \& 4$ allele, and markers of cerebral SVD (e.g., prevalent CMBs, subcortical infarcts, and WMLs). Given the underlying presumed etiopathology of CMBs by location, this suggests that pure geographic atrophy and load of retinal microvascular lesions may be useful markers of different underlying pathologies in the brain.

The association between AMD and an increased risk of clinical stroke has been reported in several population-based cohort studies. ${ }^{25,26}$ In this cohort study, we examined the longitudinal associations of retinal microvascular signs and macular degeneration with $\mathrm{CMBs}$ by anatomic location. Our findings suggest a new hypothesis that pure geographic atrophy subtype of AMD may be associated with processes also regulating cerebral neurodegeneration, insofar as CAA, which is caused by amyloid deposits in the lobar microvessels, does. ${ }^{1,4}$ It is worth noting that the prevalence of pure geographic atrophy in our sample was only $1.12 \%$, so results should be interpreted with caution.

However, the far more prevalent hypertension is the major risk factor for retinal arteriolar signs and retinopathy lesions, as well as for cerebrovascular disease ranging from clinical stroke and subclinical brain lesions (e.g., lacunar infarcts, WMLs, and CMBs) to progression of cerebral SVD, ${ }^{9,27-29}$ Our study showed that the more retinal microvascular signs present, the higher the likelihood of developing new CMBs in deep brain areas, not in strictly lobar regions. This pattern of association is in line with a hospital-based study ${ }^{30}$ that showed that retinal focal arteriolar narrowing and arteriovenous nicking were less common in patients with lobar intracerebral hemorrhage than in those with deep intracerebral hemorrhage, a sequelae of CMBs. ${ }^{1,2}$

The pathophysiologic mechanisms underlying the associations of retinal microvascular signs and geographic atrophy with region-specific CMBs are not fully understood, but studies of the etiopathophysiology of this association are emerging. First, retinal arteriolar signs and deep CMBs share common 
Table 3 Association between retinal microvascular signs and incident CMBs by anatomic location $(n=2,497)$

\begin{tabular}{|c|c|c|c|c|c|c|}
\hline \multirow[b]{2}{*}{ Retinal microvascular signs } & \multicolumn{2}{|c|}{ Global CMBs $(n=461)$} & \multicolumn{2}{|c|}{ Exclusively lobar CMBs $(n=293)^{a}$} & \multicolumn{2}{|c|}{ Deep CMBs $(n=168)^{a}$} \\
\hline & No. & Odds ratio $(95 \% \mathrm{Cl})^{\mathrm{b}}$ & No. & Odds ratio $(95 \% \mathrm{Cl})^{\mathrm{b}}$ & No. & Odds ratio $(95 \% \mathrm{Cl})^{\mathrm{b}}$ \\
\hline \multicolumn{7}{|l|}{ Focal arteriolar narrowing } \\
\hline Absence $(n=2,365)$ & 423 & 1.00 (Reference) & 272 & 1.00 (Reference) & 151 & 1.00 (Reference) \\
\hline Presence $(n=132)$ & 38 & $1.55(1.03-2.34)$ & 21 & $1.42(0.85-2.36)$ & 17 & $1.91(1.08-3.39)$ \\
\hline \multicolumn{7}{|l|}{ Arteriovenous nicking } \\
\hline Absence $(n=2,060)$ & 369 & 1.00 (Reference) & 253 & 1.00 (Reference) & 116 & 1.00 (Reference) \\
\hline Presence $(n=437)$ & 92 & $1.06(0.80-1.38)$ & 40 & $0.67(0.46-0.97)$ & 52 & $1.96(1.36-2.84)$ \\
\hline \multicolumn{7}{|l|}{ Retinopathy lesions } \\
\hline Absence $(n=2,212)$ & 392 & 1.00 (Reference) & 253 & 1.00 (Reference) & 139 & 1.00 (Reference) \\
\hline Presence $(n=285)$ & 69 & $1.33(1.00-1.88)$ & 40 & $1.26(0.86-1.79)$ & 29 & $1.49(0.98-2.27)$ \\
\hline \multicolumn{7}{|c|}{ Index of retinal microvascular signs ${ }^{c}$} \\
\hline $0(n=1,782)$ & 305 & 1.00 (Reference) & 211 & 1.00 (Reference) & 94 & 1.00 (Reference) \\
\hline $1(n=585)$ & 116 & $1.03(0.80-1.32)$ & 64 & $0.83(0.61-1.13)$ & 52 & $1.48(1.02-2.14)$ \\
\hline$\geq 2(n=130)$ & 40 & $1.76(1.16-2.69)$ & 18 & $1.21(0.70-2.09)$ & 22 & $3.00(1.73-5.20)$ \\
\hline$p$ for trend & & 0.047 & & 0.760 & & $<0.001$ \\
\hline
\end{tabular}

Abbreviations: $\mathrm{Cl}=$ confidence interval; $\mathrm{CMB}=$ cerebral microbleed.

a Participants with no incident CMBs $(n=2,036)$ were held as a referent group for multinomial logistic modeling when the associations with incident $C M B s$ by anatomic location (i.e., exclusively lobar or deep CMBs vs no CMBs) were examined.

b Odds ratio $(95 \% \mathrm{Cl})$ was adjusted for age, sex, types of coils, follow-up interval, current smoking, systolic blood pressure, use of antihypertensive drugs, body mass index, diabetes mellitus, total cholesterol, baseline prevalent CMBs, subcortical infarcts, volume of white matter lesions, and APOE $\varepsilon 4$ allele.

'The composite index (range 0-3) was computed by counting the 3 retinal microvascular signs (i.e., retinal focal arteriolar narrowing, arteriovenous nicking, and retinopathy lesions) concurrently present in the participant.

cardiovascular risk factors, especially hypertension. ${ }^{6}$ Second, disruption of the blood-retina barrier from retina hypoxia owing to long-term exposures to cardiovascular risk factors, which is analogous to and correlated with the blood-brain barrier dysfunction, may play a part in the pathogenesis of both retinal microvascular lesions and cerebral microangiopathy. ${ }^{30,31} \mathrm{Fi}-$ nally, evidence has emerged that $\beta$-amyloid, as a hallmark of Alzheimer pathology and CAA in the brain, ${ }^{32,33}$ also exists in the retina and the heart of patients with $\mathrm{AD}^{34-36}$ Given the homology of microvasculature in the brain and the retina and the involvement of $\beta$-amyloid in both retinal degeneration (AMD) and CAA, ${ }^{10,12,37,38}$ the link of pure geographic atrophy with strictly lobar CMBs may reflect underlying pathologic processes common in both the brain and the retina. Given that experimental and clinical studies have shown amyloid deposits in the retina, the hypothesis of neurodegenerative-like process in AMD is of interest to follow up in populations in which the prevalence of pure geographic atrophy or, in reverse, $\mathrm{AD}$ is higher than in our cohort.

Strengths of this study include the prospective nature of the study design, the community-derived large cohort of older adults, and control of multiple potential confounders, including markers of cerebral SVD. Furthermore, the MRI sequences and definition of CMBs used in our study were comparable to those in the recommended standard scales (e.g., Microbleed Anatomical
Rating Scale [MARS] and Brain Observer MicroBleed Scale [BOMBS] $).{ }^{1}$ Our study also has limitations. First, the analytic sample was younger and healthier than those excluded, which may potentially bias our estimates. Second, a potential limitation of the retinal microvascular index was that the 3 retinal microvascular signs might reflect different stages of retinal microvascular alterations. However, the composite index of retinal microvascular profile may be more stable than the individual retinal makers because single retinal microvascular signs may fluctuate as a result of transient metabolic and hemodynamic alterations. ${ }^{39}$ Finally, statistical power was limited for the analysis of certain subgroups (e.g., deep CMBs in late AMD).

Overall, our findings suggest that additional studies are necessary to explore common pathophysiologic processes of microvascular damage and neurodegeneration in the retina and the brain. In combination with additional biological and functional information, it may be possible in the future to develop retinal screening algorithms for brain disease and to identify elderly people at high risk for $\mathrm{CMBs}$ and related functional consequences.

\section{Author contributions}

Study design: C.Q. J.D., V.G., M.F.C., L.J.L. Data collection: S.S., G.E., R.K., M.A.v.B., V.G. Data analysis: J.D., D.E.F., Q.Z. 
Data interpretation: all authors. Drafting of the manuscript: C.Q. J.D., L.J.L. Critical revision of the manuscript: all authors.

\section{Acknowledgment}

The authors thank all the participants of the AGES-Reykjavik Study and the Icelandic Heart Association clinic staff for their invaluable contributions.

\section{Study funding}

The AGES-Reykjavik Study was funded by the NIH (contract N01-AG-12100); the Intramural Research Program of the National Institute on Aging and the National Eye Institute (ZIAEY000401), NIH; and the Icelandic Heart Association and the Icelandic Parliament. None of the funding organizations or sponsors were involved in study design; in the collection, analysis, or interpretation of data; in writing of the report; or in the decision to submit the manuscript for publication.

\section{Disclosure}

C. Qiu was supported by grants from the Swedish Research Council (2015-2531) and the Swedish Research Council for Health, Working Life and Welfare (2014-1382), Stockholm, Sweden. J. Ding, S. Sigurdsson, D. Fisher, Q. Zhang, G. Eiriksdottir, R. Klein, M.A. van Buchem, and V. Gudnason report no disclosures relevant to the manuscript. M. Cotch is on the editorial boards of the journals Ophthalmology and Ophthalmic Epidemiology. L. Launer reports no disclosures relevant to the manuscript. Go to Neurology.org/ $\mathrm{N}$ for full disclosures.

Received February 17, 2017. Accepted in final form September 28, 2017.

\section{References}

1. Greenberg SM, Vernooij MW, Cordonnier C, et al. Cerebral microbleeds: a guide to detection and interpretation. Lancet Neurol 2009;8:165-174

2. Akoudad S, Portegies ML, Koudstaal PJ, et al. Cerebral microbleeds are associated with an increased risk of stroke: the Rotterdam Study. Circulation 2015;132:509-516.

3. Akoudad S, Wolters FJ, Viswanathan A, et al. Association of cerebral microbleeds with cognitive decline and dementia. JAMA Neurol 2016;73:934-943.

4. Wardlaw JM, Smith EE, Biessels GJ, et al. Neuroimaging standards for research into small vessel disease and its contribution to ageing and neurodegeneration. Lancet Neurol 2013;12:822-838.

5. London A, Benhar I, Schwartz M. The retina as a window to the brain: from eye research to CNS disorders. Nat Rev Neurol 2013;9:44-53.

6. Cheung CY, Ikram MK, Sabanayagam C, Wong TY. Retinal microvasculature as a model to study the manifestations of hypertension. Hypertension 2012;60: 1094-1103.

7. Cheung N, Mosley T, Islam A, et al. Retinal microvascular abnormalities and subclinical magnetic resonance imaging brain infarct: a prospective study. Brain 2010; 133:1987-1993.

8. Kawasaki R, Cheung N, Mosley T, et al. Retinal microvascular signs and 10-year risk of cerebral atrophy: the Atherosclerosis Risk in Communities (ARIC) study. Stroke 2010;41:1826-1828.

9. Hanff TC, Sharrett AR, Mosley TH, et al. Retinal microvascular abnormalities predict progression of brain microvascular disease: an Atherosclerosis Risk in Communities Magnetic Resonance Imaging Study. Stroke 2014;45:1012-1017.

10. Ratnayaka JA, Serpell LC, Lotery AJ. Dementia of the eye: the role of amyloid beta in retinal degeneration. Eye (Lond) 2015;29:1013-1026.
11. den Haan J, Janssen SF, van Berckel BN, Teunissen CE, Verbraak F, Bouwman FH. Retinal thickness in Alzheimer's disease: a potential diagnostic biomarker? Evidence from an amyloid proven early onset Alzheimer's disease cohort. Alzheimers Dement 2016;12(suppl):678-679.

12. Koronyo-Hamaoui M, Koronyo Y, Ljubimov AV, et al. Identification of amyloid plaques in retinas from Alzheimer's patients and noninvasive in vivo optical imaging of retinal plaques in a mouse model. Neuroimage 2011;54(suppl 1):S204-S217.

13. Laske C, Sohrabi HR, Frost SM, et al. Innovative diagnostic tools for early detection of Alzheimer's disease. Alzheimers Dement 2015;11:561-578.

14. Campbell MC, Kisilak ML, DeVries D, et al. Amyloid as a biomarker of Alzheimer's disease in post-mortem retinas in human and dog models of Alzheimer's disease. Alzheimers Dement 2016;12(suppl):319-320.

15. Qiu C, Cotch MF, Sigurdsson S, et al. Retinal and cerebral microvascular signs and diabetes: the AGES-Reykjavik Study. Diabetes 2008;57:1645-1650.

16. Qiu C, Cotch MF, Sigurdsson S, et al. Cerebral microbleeds and age-related macular degeneration: the AGES-Reykjavik Study. Neurobiol Aging 2012;33:2935-2937.

17. Harris T, Launer LJ, Eiriksdottir G, et al. Age, Gene/Environment Susceptibility-Reykjavik Study: multidisciplinary applied phenomics. Am J Epidemiol 2007;165:1076-1087.

18. Ding J, Sigurdsson S, Garcia M, et al. Risk factors associated with incident cerebral microbleeds according to location in older people: the Age, Gene/Environment Susceptibility (AGES)-Reykjavik Study. JAMA Neurol 2015;72:682-688.

19. Jonasson F, Arnarsson A, Eiríksdottir G, et al. Prevalence of age-related macular degeneration in old persons: Age, Gene/Environment Susceptibility Reykjavik Study. Ophthalmology 2011;118:825-830.

20. Klein R, Davis MD, Magli YL, Segal P, Klein BE, Hubbard L. The Wisconsin agerelated maculopathy grading system. Ophthalmology 1991;98:1128-1134.

21. Qiu C, Cotch MF, Sigurdsson S, et al. Microvascular lesions in the brain and retina: the AGES-Reykjavik Study. Ann Neurol 2009;65:569-576.

22. Sigurdsson S, Aspelund T, Forsberg L, et al. Brain tissue volumes in the general population of the elderly: the AGES-Reykjavik Study. Neuroimage 2012;59: $3862-3870$.

23. Hafsteinsdottir SH, Eiriksdottir G, Sigurdsson S, et al. Brain tissue volumes by APOE genotype and leisure activity: the AGES-Reykjavik Study. Neurobiol Aging 2012;33 829.e1-829.e8.

24. Kim DH, Newman $\mathrm{AB}$, Hajjar I, et al. Retinal microvascular signs and functional loss in older persons: the Cardiovascular Health Study. Stroke 2011;42:1589-1595.

25. Wieberdink RG, Ho L, Ikram MK, et al. Age-related macular degeneration and the risk of stroke: the Rotterdam Study. Stroke 2011;42:2138-2142.

26. Ikram MK, Mitchell P, Klein R, Sharrett AR, Couper DJ, Wong TY. Age-related macular degeneration and long-term risk of stroke subtypes. Stroke 2012;43: 1681-1683.

27. Doubal FN, Hokke PE, Wardlaw JM. Retinal microvascular abnormalities and stroke: a systematic review. J Neurol Neurosurg Psychiatry 2009;80:158-165.

28. Heringa SM, Bouvy WH, van den Berg E, Moll AC, Kappelle LJ, Biessels GJ. Associations between retinal microvascular changes and dementia, cognitive functioning, and brain imaging abnormalities: a systematic review. J Cereb Blood Flow Metab 2013;33:983-995.

29. Kawasaki R, Xie J, Cheung N, et al. Retinal microvascular signs and risk of stroke: the Multi-Ethnic Study of Atherosclerosis (MESA). Stroke 2012;43:3245-3251.

30. Baker ML, Hand PJ, Wong TY, et al. Retinopathy and lobar intracerebral hemorrhage: insights into pathogenesis. Arch Neurol 2010;67:1224-1230.

31. Kaur C, Foulds WS, Ling EA. Blood-retinal barrier in hypoxic ischaemic conditions: basic concepts, clinical features and management. Prog Retin Eye Res 2008;27:622-647.

32. Gurol ME, Irizarry MC, Smith EE, et al. Plasma beta-amyloid and white matter lesions in $\mathrm{AD}, \mathrm{MCI}$, and cerebral amyloid angiopathy. Neurology 2006;66:23-29.

33. Koyama A, Okereke OI, Yang T, Blacker D, Selkoe DJ, Grodstein F. Plasma amyloid- $\beta$ as a predictor of dementia and cognitive decline: a systematic review and metaanalysis. Arch Neurol 2012;69:824-831.

34. Guymer R, Cipriani T, Rittenhouse KD, et al. Plasma levels of amyloid beta and other proinflammatory mediators in patients with age-related macular degeneration. Graefes Arch Clin Exp Ophthalmol 2015;253:1347-1354.

35. Shah TM, Gupta SM, Chatterjee P, Campbell M, Martins RN. Beta-amyloid sequelae in the eye: a critical review on its diagnostic significance and clinical relevance in Alzheimer's disease. Mol Psychiatry 2017;22:353-363.

36. Troncone $\mathrm{L}$, Luciani $\mathrm{M}$, Coggins $\mathrm{M}$, et al. $\mathrm{A} \beta$ amyloid pathology affects the hearts of patients with Alzheimer's disease: mind the heart. J Am Coll Cardiol 2016;68: 2395-2407.

37. Ohno-Matsui K. Parallel findings in age-related macular degeneration and Alzheimer's disease. Prog Retin Eye Res 2011;30:217-238.

38. Sivak JM. The aging eye: common degenerative mechanisms between the Alzheimer's brain and retinal disease. Invest Ophthalmol Vis Sci 2013;54:871-880.

39. Belsky DW, Caspi A, Houts R, et al. Quantification of biological aging in young adults. Proc Natl Acad Sci USA 2015;112:E4104-E4110. 


\title{
Differential associations between retinal signs and $\mathrm{CMBs}$ by location
}

\author{
The AGES-Reykjavik Study
}

Chengxuan Qiu, PhD, Jie Ding, PhD, Sigurdur Sigurdsson, MSc, Diana E. Fisher, MA, Qian Zhang, MSc, Gudny Eiriksdottir, MSc, Ronald Klein, MD, Mark A. van Buchem, MD, PhD, Vilmundur Gudnason, MD, PhD, Mary Frances Cotch, PhD, and Lenore J. Launer, PhD

Neurology ${ }^{\circledR}$ 2018;90:63. doi:10.1212/WNL.0000000000004792

\author{
Correspondence \\ Dr. Launer \\ launerl@nia.nih.gov \\ or Dr. Qiu \\ chengxuan.qiu@ki.se
}

\section{Study question}

Are age-related macular degeneration (AMD) and retinal microvascular signs differentially associated with lobar and deep cerebral microbleeds ( $\mathrm{CMBs})$ ?

\section{Summary answer}

In older people, retinal microvascular signs are associated with deep CMBs. Pure geographic atrophy, a form of AMD, is associated exclusively with lobar CMBs.

\section{What is known and what this paper adds}

Retinal microvascular signs are associated with brain lesions and infarcts. AMD is a potential neurodegenerative biomarker. We elucidated how AMD and retinal microvascular signs are associated with $\mathrm{CMBs}$ in specific brain areas.

\section{Participants and setting}

We examined 2,502 Icelandic Age, Gene/Environment Susceptibility (AGES)-Reykjavik Study participants born in 1907 to 1935 .

\section{Design, size, and duration}

Participants were examined in 2002 to 2006 and 2007 to 2011. Both examinations included brain MRI scans, which permitted CMB assessments, and fundus photography, which permitted assessments of AMD and retinal microvascular signs (i.e., focal arteriolar narrowing, arteriovenous nicking, and retinopathic lesions). Collected images were analyzed automatically or by evaluators blinded to participant identities. Binomial logistic models were used to calculate odds ratios (ORs) for CMBs, and multinomial logistic models were used to assess how AMD and retinal microvascular signs were associated with $\mathrm{CMB}$ locations.

\section{Main results and the role of chance}

Over the follow-up period (mean $5.2 \pm 0.2$ years), new CMBs were detected in 461 (18.43\%) participants. Pure

\begin{tabular}{lll}
\hline & $\begin{array}{l}\text { OR }(95 \% \mathrm{Cl}) \text { for } \\
\text { exclusively lobar CMBs }\end{array}$ & $\begin{array}{l}\text { OR }(95 \% \mathrm{Cl}) \text { for } \\
\text { deep CMBs }\end{array}$ \\
\hline Early AMD vs no AMD & $0.96(0.68-1.34)$ & $0.82(0.53-1.26)$ \\
\hline Late AMD vs no AMD & $1.40(0.76-2.56)$ & $0.34(0.10-1.16)$ \\
\hline $\begin{array}{l}\text { Presence of a retinal } \\
\text { microvascular sign }\end{array}$ & $0.83(0.61-1.13)$ & $1.48(1.02-2.14)$ \\
\hline
\end{tabular}

geographic atrophy was associated with increased risk of new CMBs (OR 1.91, 95\% confidence interval [CI] $0.80-4.56$ ), particularly exclusively lobar CMBs (OR 2.59, 95\% CI 1.01-6.65). Each additional observed retinal microvascular sign was associated with increased risk of new CMBs ( $p$ for trend $=0.047$ ), especially deep CMBs ( $p$ for trend $<0.001)$.

\section{Bias, confounding, and other reasons for caution}

The low prevalence of pure geographic atrophy noted $(1.12 \%)$ is a reason for caution. The participants included were younger and healthier than the excluded participants. The 3 analyzed retinal microvascular signs may reflect different stages of retinal microvascular alterations. Statistical power was limited for some subgroup analyses.

\section{Generalizability to other populations}

Because we examined a large and well-established cohort, the results are probably generalizable to similar populations.

\section{Study funding/potential competing interests}

This study was supported by the NIH, the Icelandic Heart Association, and the Icelandic government. Dr. Cotch serves on the editorial boards of 2 journals. Dr. Qiu has received Swedish Research Council grants. Go to Neurology.org/N for full disclosures. 


\section{Neurology}

\section{Differential associations between retinal signs and CMBs by location: The AGES-Reykjavik Study \\ Chengxuan Qiu, Jie Ding, Sigurdur Sigurdsson, et al.}

Neurology 2018;90;e142-e148 Published Online before print December 13, 2017

DOI 10.1212/WNL.0000000000004792

\section{This information is current as of December 13, 2017}

\section{Updated Information \&} Services

References

Subspecialty Collections

Permissions \& Licensing

Reprints including high resolution figures, can be found at: http://n.neurology.org/content/90/2/e142.full

This article cites 39 articles, 13 of which you can access for free at: http://n.neurology.org/content/90/2/e142.full\#ref-list-1

This article, along with others on similar topics, appears in the following collection(s):

Cohort studies

http://n.neurology.org/cgi/collection/cohort_studies

MRI

http://n.neurology.org/cgi/collection/mri

Retina

http://n.neurology.org/cgi/collection/retina

Information about reproducing this article in parts (figures,tables) or in its entirety can be found online at:

http://www.neurology.org/about/about_the_journal\#permissions

Information about ordering reprints can be found online:

http://n.neurology.org/subscribers/advertise

Neurology ${ }^{\circledR}$ is the official journal of the American Academy of Neurology. Published continuously since 1951, it is now a weekly with 48 issues per year. Copyright Copyright ( 2017 The Author(s). Published by Wolters Kluwer Health, Inc. on behalf of the American Academy of Neurology.. All rights reserved. Print ISSN: 0028-3878. Online ISSN: 1526-632X.

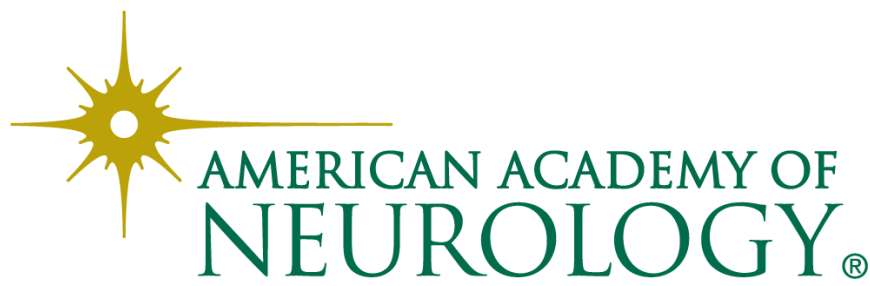

